\title{
Gender differences in major vascular complications of catheter ablation for atrial fibrillation
}

\author{
Jiř́i Plášek ${ }^{1}$, Dan Wichterle ${ }^{2}$, Petr Peichl ${ }^{3}$, Robert Čihák ${ }^{1}$, Patrik Jarkovský ${ }^{4}$, Tomáš \\ Roubíček $^{5}$, Predrag Stojadinović ${ }^{1}$, Jana Hašková ${ }^{1}$, and Josef Kautzner ${ }^{6}$ \\ ${ }^{1}$ Institut klinicke a experimentalni mediciny \\ ${ }^{2}$ General University Hospital \\ ${ }^{3}$ IKEM \\ ${ }^{4}$ Ústřední vojenská nemocnice - Vojenská fakultní nemocnice Praha \\ ${ }^{5}$ Krajská nemocnice Liberec as \\ ${ }^{6}$ Institute for Clinical and Experimental Medicine
}

September 28, 2020

\begin{abstract}
Aims: Catheter ablation ( $\mathrm{CA}$ ) for atrial fibrillation (AF) has a considerable risk of procedural complications. Major vascular complications (MVCs) appear to be the most frequent. This study investigated gender differences in MVCs in patients undergoing CA for AF. Methods: A total of 4734 CAs for AF (65\% paroxysmal, $26 \%$ repeated procedures) were performed at our center between January 2006 and August 2018. Patients (71\% males) aged $60 \pm 10$ years and had body mass index of $29 \pm 4$ $\mathrm{kg} / \mathrm{m} 2$. Radiofrequency point-by-point ablation was employed in $96.3 \%$ procedures with the use of $3 \mathrm{D}$ navigation systems and facilitated by intracardiac echocardiography. Pulmonary vein isolation was mandatory; cavotricuspid isthmus and left atrial substrate ablation were performed in $22 \%$ and $38 \%$ procedures, respectively. MVCs were defined as those that resulted in permanent injury, required intervention, or prolonged hospitalization. Their rates and risk factors were compared between genders. Results: A total of $112(2.4 \%)$ MVCs were detected: 54/1512 (3.5\%) in females and 58/3222 (1.8\%) in males (P<0.0001). On multivariate analysis, lower body height was the only risk factor for MVCs in females $(\mathrm{P}=0.0005)$. On the contrary, advanced age was associated with MVCs in males $(\mathrm{P}=0.006)$. Conclusion: Females have a higher risk of MVCs following CA for AF compared to males. This difference is driven by lower body size in females. Low body height in females and advanced age in males are independent predictors of MVCs. US-guided venipuncture lowered the MVC rate in males.
\end{abstract}

\section{Gender differences in major vascular complications of catheter ablation for atrial fibrillation}

Jiří Plášek ${ }^{1,2,6}$, Dan Wichterle ${ }^{1,3}$, Petr Peichl ${ }^{1}$, Robert Čihák ${ }^{1}$, Patrik Jarkovský1,4, Tomáš Roubíček ${ }^{1,5}$, Predrag Stojadinović ${ }^{1}$, Jana Hašková ${ }^{1}$, Josef Kautzner ${ }^{1,6}$

${ }^{1}$ Dept. of Cardiology, Institute for Clinical and Experimental Medicine, Prague, Czech Republic

${ }^{2}$ Dept. of Cardiology, University Hospital Ostrava and Medical School, University of Ostrava, Czech Republic

${ }^{3}$ Dept. of Cardiovascular Medicine, $1^{\text {st }}$ Medical School, Charles University and General University Hospital in Prague, Prague, Czech Republic

${ }^{4}$ Dept. of Cardiology, Military University Hospital Prague, Czech Republic

${ }^{5}$ Dept. of Cardiology, Regional Hospital Liberec, Czech Republic

${ }^{6}$ Dept. of Internal Medicine I, Palacky University Hospital, Olomouc, Czech Republic 


\section{Corresponding author:}

Dr Jiří Plášek

Dept. of Cardiology

Institute for Clinical and Experimental Medicine (IKEM)

Vídeňská 1958/9, Prague, 140 21, Czech Republic

Tel: +420236055006

Fax: +420236052985

E-mail: jiri.plasek@ikem.cz

Word count: 3356

\section{Abstract}

Aims: Catheter ablation (CA) for atrial fibrillation (AF) has a considerable risk of procedural complications. Major vascular complications (MVCs) appear to be the most frequent. This study investigated gender differences in MVCs in patients undergoing CA for AF in a high-volume tertiary center.

Methods: A total of 4734 CAs for AF (65\% paroxysmal, $26 \%$ repeated procedures) were performed at our center between January 2006 and August 2018. Patients (71\% males) aged $60 \pm 10$ years and had body mass index of $29 \pm 4 \mathrm{~kg} / \mathrm{m}^{2}$ at the time of the procedure. Radiofrequency point-by-point ablation was employed in $96.3 \%$ procedures with the use of 3D navigation systems and facilitated by intracardiac echocardiography. Pulmonary vein isolation was mandatory; cavotricuspid isthmus and left atrial substrate ablation were performed in $22 \%$ and $38 \%$ procedures, respectively. MVCs were defined as those that resulted in permanent injury, required intervention, or prolonged hospitalization. Their rates and risk factors were compared between genders.

Results : A total of $112(2.4 \%)$ MVCs were detected: 54/1512 (3.5\%) in females and 58/3222 (1.8\%) in males $(\mathrm{P}<0.0001)$. On multivariate analysis, lower body height was the only risk factor for MVCs in females $(\mathrm{P}=0.0005)$. On the contrary, advanced age was associated with MVCs in males $(\mathrm{P}=0.006)$.

Conclusion: Females have a higher risk of MVCs following CA for AF compared to males. This difference is driven by lower body size in females. Low body height in females and advanced age in males are independent predictors of MVCs. US-guided venipuncture lowered the MVC rate in males.

Keywords: atrial fibrillation; catheter; ablation; vascular; complications; risk; US-guided venipuncture.

\section{Introduction}

Atrial fibrillation (AF) is the most common arrhythmia with an incidence of $2 \%$ in the unselected population; it increases dramatically with age and comorbidities. ${ }^{1}$ Catheter ablation $(\mathrm{CA})$ is an established rhythm control treatment modality for drug-refractory symptomatic $\mathrm{AF}$ or as a patient-preferred treatment approach. However, the risk of procedural complications may not be negligible. Recently, the risk of major complications from the population-based cohort was reported ranging from 3.8 to $7.2 \%{ }^{2}$ Female gender was identified as a risk factor for complications in several studies. ${ }^{3,4}$ While major non-vascular complications are highly heterogeneous and rather unpredictable, major vascular complications (MVCs) have, in part, common pathogenesis, and their associations with clinical and procedural characteristics deserve to be investigated. The goal of this study was to analyze this separately in males and females.

\section{Methods}

All patients undergoing CA for AF at our institution between January 2006 and August 2018 were analyzed including repeated procedures for AF and/or left-sided atrial tachycardias. All patients signed informed 
consent about the procedure. The study was approved by the institutional review board and conducted according to the Helsinki declaration. The need for informed consent was waived for the study.

Ablation procedure

Bilateral femoral veins were punctured either using anatomical landmarks or under direct ultrasound (US) guidance (from 2016). In the most common setting, two Swartz SL1 8.5 Fr sheaths were introduced via the right femoral vein and two short sheaths via the left femoral vein: $7 \mathrm{Fr}$ for coronary sinus catheter and $11 \mathrm{Fr}$ for intracardiac echocardiography probe. Between 2006 and 2012, the right jugular / subclavian access was used for coronary sinus catheter placement. For robotic cases (Sensei, Hansen Medical, Mountain View, CA, USA), which were performed between 2008 and 2011, 14 Fr sheath was employed to accommodate Artisan electromechanical catheter from the right femoral vein. No arterial access was used for procedure monitoring. In the early period, sheaths were removed after activated clotting time (ACT) level dropped below $250 \mathrm{~s}$ and the puncture sites were compressed for 6 hours. Since 2014, the sheaths were removed at the end of the procedure and venous hemostasis was achieved by "Z'-stitch. Bed rest was implemented in all patients till the next morning.

Intracardiac echocardiography was used throughout the whole procedure as an institutional standard. It was used particularly for guiding the transseptal puncture, determining accurate antral ablation points, tagging the course of the esophagus, titration of radiofrequency energy, and early detection of complications.

CA was performed with a $3.5 \mathrm{~mm}$ irrigated-tip catheter Navistar $^{(\mathrm{r})}$ Thermocool or Thermocool Smarttouch $^{(\mathrm{r})}$ or Celsius $^{(\mathrm{r})}$ Thermocool; Biosense Webster, Diamond Bar, CA, USA). The catheter was navigated with the use of 3D electroanatomic system $\left(\mathrm{CARTO}^{(\mathrm{r})}\right.$; Biosense Webster or Ensite NavX ${ }^{(\mathrm{r})}$; St Jude Medical, St Paul, MN, USA). Linear point-by-point lesions were placed around the ostia of pulmonary veins with the endpoint of electrical isolation. In patients with the advanced atrial disease (38\%), empirical linear lesions and/or superior vena cava isolation and/or biatrial electrogram-guided ablation was performed. Cavotricuspid isthmus was ablated in $22 \%$ of procedures. Mappable ATs were always targeted. Non-inducibility of arrhythmia was the desired endpoint of repeated procedures.

Radiofrequency energy was delivered by an EP Shuttle(Stockert, Freiburg, Germany) or Smartablate(Biosense Webster) or Ampere generator (St Jude Medical). Constant irrigation flow of $15 \mathrm{ml} / \mathrm{min}$ $\left(30 \mathrm{ml} / \mathrm{min}\right.$ inside the coronary sinus) through a Cool Flow ${ }^{(\mathrm{r})}$ pump (Biosense Webster) or Cool Point (St Jude Medical), respectively, was employed. The power mode was used with a preset power up to $25-35 \mathrm{~W}$ and down-regulation when the tip temperature of $43 \mathrm{degC}$ was achieved. Power output was mostly reduced to $20-25 \mathrm{~W}$ at the left atrium posterior wall and inside the coronary sinus. The energy was delivered either in point-by-point fashion (20-30 s at one spot) or by dragging. Rarely $(<5 \%)$ other ablation catheters were

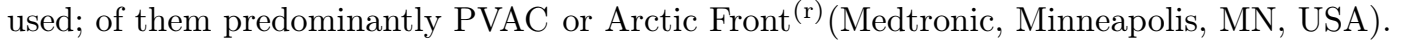

\section{Perioperative anticoagulation}

In the early period from 2006 to 2012, warfarin was temporarily discontinued to achieve International Normalized Ratio (INR) $<2$ and bridged with weight-adjusted low molecular weight heparin (LMWH). At the beginning of every procedure, unfractionated heparin was administered with a loading dose of $5000 \mathrm{IU}$ before the first transseptal puncture. The ACT was measured every 15-30 minutes, with the target value of 300-350 s in all patients during the procedure. After the procedure, the infusion of unfractionated heparin was administered until the morning of the first post-ablation day. Subsequently, the warfarin was restarted and LMWH administered until reaching the therapeutic level of INR.

From 2013, all procedures were performed on uninterrupted warfarin with target INR between 2 and 3 or minimally (single dose) interrupted direct oral anticoagulants. After the procedure, either direct oral anticoagulants or warfarin were re-started after the drop of the ACT under $170 \mathrm{~s}$.

Anticoagulation treatment continued for at least 3 months after the procedure in low-risk patients or lifelong in high-risk patients according to the guidelines. ${ }^{5}$ 


\section{Assessment of complications}

The specific institutional tracking system was used to identify all complications during and after the procedure until the 3-month clinical outpatient visit. This included a purpose-established central registry for complications of invasive procedures, which was described elsewhere. ${ }^{6}$ All recorded complications were individually reviewed by staff physicians of the arrhythmia service in morbidity and mortality meetings and classified as vascular or other, and major or minor.

\section{Definition of complications}

Major complications were defined as those that result in permanent injury or death, require intervention for treatment, prolong $\left(>48 \mathrm{~h}\right.$ ) hospitalization or require new hospitalization. ${ }^{6}$ MVCs were major complications limited to vascular access. Hemoglobin drop by $>30 \mathrm{~g} / \mathrm{l}$ was classified as MVC even if it was not treated with blood transfusion.

\section{Statistical analysis}

Continuous variables were expressed as means +- standard deviation and compared by t-test or MannWhitney U test, as appropriate. Categorical variables are expressed as percentages and compared by the chi-square test, Fisher exact test or logistic regression if appropriate. Association of baseline clinical and procedural factors with MVCs was investigated by linear regression analysis. Statistically significant factors on univariate analysis $(\mathrm{P}<0.1)$ were entered into a multivariate regression model. Two-tailed $\alpha<0.05$ was considered statistically significant, except for the test of equality of covariance matrices where $\mathrm{P}<0.005$ was considered significant. For post hoc comparison of subgroups, Tukey HSD or Bonferroni test was used where appropriate. The majority of analyses were performed separately for males and females. All analyses were performed using TIBCO Statistica version 13.3 (Palo Alto, CA, USA) or IBM SPSS for MAC version 23 (IBM, New York, USA).

\section{Results}

A total of $4734 \mathrm{CAs}$ were analyzed. Baseline clinical and procedural characteristics (per procedure assessment) including gender differences are shown in Table 1. There were no meaningful differences in the medication between males and females. Overall, 188 (4.0\%) major complications were detected: $86 / 1512$ $(5.7 \%)$ in females and $102 / 3222(3.2 \%)$ in males $(\mathrm{P}<0.0001)$. Of them, $59.6 \%$ were classified as MVCs. A total of $112(2.4 \%)$ MVCs were unevenly distributed between genders: 54/1512 (3.5\%) in females and 58/3222 $(1.8 \%)$ in males $(\mathrm{P}<0.0001)$. Thrombin injection, blood transfusion or surgical revision was required in $45 \%$ of all MVCs. There was a significant trend in the reduction of MVCs during the study period (Figure 1) with an average year-to-year drop by $0.13 \%(\mathrm{P}=0.048)$, which produced an overall drop by $1.7 \%$ during the study span of almost 13 years. Several clinical and procedural characteristics differed between those with and without MVCs as shown separately for males and females in Table 2. Details on gender differences in the frequency of individual MVCs are provided in Table 3. The female gender was associated with relative risk (RR) of 3.2 (95\% confidence interval (CI): 1.9-5.4, P $<0.0001)$ of developing groin hematoma, RR of 3.9 (95\% CI: $1.9-7.9, \mathrm{P}<0.0001)$ of significant drop of hemoglobin level, $\mathrm{RR}$ of $2.6(95 \% \mathrm{CI}: 1.1-6.7, \mathrm{P}=$ 0.02 ) of blood transfusion, and RR of 2.3 (95\% CI: $1.1-5.1, \mathrm{P}=0.02)$ of surgical revision.

\section{Predictors of MVCs}

Among "modifiable" factors, the strategy of non-interrupted anticoagulation and "Z"-stitch for hemostasis did not appear to have a significant impact on the reduction of the rate of MVCs (Table 2). On the contrary, US-guided venipuncture was associated with 1.44\% drop of MVCs in males but not in females.

Longer procedures were associated with a higher rate of MVCs with an increase of $0.21 \%$ and $0.39 \%$ per every 30 minutes of procedure duration in males and females, respectively. The rate of MVCs slowly decreased by $0.15 \%$ and $0.12 \%$ per year in males and females, respectively, during the study period. Both relationships were statistically significant only in males (Table 4 ). 
The risk of MVCs almost linearly rose with age in both males and females (Figure 2). On univariate regression analysis, older age, and lower body height were associated with MVCs in females (Table 4). Particularly, low-stature women $(<170 \mathrm{~cm}$ ), were at the highest risk of MVCs (Figure 3). In males, older age, smaller body surface area (BSA), persistent AF, and longer procedure time was associated with MVCs (Table 4). Interestingly, the body weight was almost unrelated to MVCs in both genders (Table 4, Figure 3). The combined impact of body height and weight is illustrated separately for females and males in Figure 4.

On multivariate regression analysis in males (Table 4), age and US-guided venipuncture were independently associated with MVC that increased by $0.64 \%$ per every decade of age and, on the other hand, decreased by $1.55 \%$ when US-guided venous access was employed. The same analysis in females revealed that only body height was independently associated with MVCs that increased by $1.3 \%$ per 5 -cm decrease of body height.

When the multivariate analysis was performed in the total cohort, age $(\mathrm{P}=0.002)$, body height $(\mathrm{P}=0.0002)$, and year of procedure $(\mathrm{P}=0.01)$ were associated with the rate of MVCs, while gender itself was not an independent risk factor $(\mathrm{P}=0.54)$.

\section{Discussion}

The main findings of our retrospective analysis can be summarized as follows: 1) MVCs were the most frequent among major complications of $\mathrm{CA}$ for $\mathrm{AF}$ and occurred twice as often in females than in males, 2) Body size, particularly low body height in females, was an important determinant of MVCs, 3) US-guided venipuncture lowered MVCs only in males, and 4) trend in reducing MVCs was observed during the study period.

Risk of vascular complications

The above data on a higher frequency of vascular and bleeding complications in females after catheter ablation for AF (including hospital readmissions) were recently reported from the US nationwide database, analyzing nearly 55 thousand patients. ${ }^{7}$ However, no subgroup analysis was performed for anthropometric parameters in this study. Another recent analysis of 21 thousand patients with the previous ablation for AF confirmed that females have a higher risk of virtually all complications and more frequent readmissions. ${ }^{8}$ Both Shah et al. ${ }^{4}$ and Spragg et al. ${ }^{9}$ identified female gender (odds ratio (OR): 1.3, 95\% CI: 1.1-1.7, P $<0.05$ and OR: 3.0, 95\% CI: $1.3-7.2, \mathrm{P}=0.014$, respectively) as the independent predictors of major complications of the $\mathrm{AF}$ ablation procedure. In the latter series, the vascular injury was the most prevalent complication, occurring in $1.7 \%$ of the cases. ${ }^{9}$ In other similar studies, access site hematoma was by far the most common vascular complication which was more frequent in females vs. males $(2.1$ vs. $0.7 \%$ and 6.8 vs. $0.9 \%, \mathrm{P}=$ $0.026, \mathrm{P}=0.027$ respectively). ${ }^{10,11}$ All the above data are very similar to our results, where groin hematoma accounted for 2.4 and $0.7 \%$ in females and males, respectively. In total, MVCs in our trial reached $2.4 \%$ and included also groin hematomas prolonging hospitalization and/or retroperitoneal bleeding. This rate may look higher than in other studies, where vascular complications ranged from 1.1 to $1.9 \%{ }^{12,13}$ However, these trials mostly addressed only arterio-venous fistula and pseudoaneurysm as vascular complications. In a trial by Shah et al. ${ }^{4}$, in which vascular complications were classified more broadly, the vascular complication rate reached $2.6 \%$, replicating our results.

Vascular complications are also the most common in patients undergoing percutaneous coronary interventions with access site hematoma the most frequent among them. ${ }^{14}$ Even in this setting of arterial access and the use of antiplatelet drugs together with unfractionated heparin, bleeding complications were more pronounced in females vs. males (4.3 vs. $1.9 \%$ with OR: $2.3(95 \% \text { CI: } 1.6-3.3)^{14}$ or 5.8 vs. $2.5 \%,(\mathrm{P}=0.02) .{ }^{15}$

Summarizing all the above data, both $\mathrm{CA}$ for $\mathrm{AF}$ and percutaneous coronary intervention bear a similar rate of vascular complications, which are higher in females compared to males.

Body size as a risk factor

Searching for predictors of MVCs after CA for AF, we revealed an important role of body size. The body size can be characterized by body weight or height or BSA. Of these, the body height was most tightly 
associated with MVCs although BSA was a significant competitor that was comparably strong in prediction models. In our previous study, low body weight was identified as a single independent risk factor for major complications with a $0.8 \%$ increase per $10 \mathrm{~kg}$ of body weight reduction. ${ }^{6}$ This analysis covered the early period when oral anticoagulants were interrupted before the procedure and low molecular weight bridging was used. Such a strategy could contribute to a higher risk of bleeding in subjects with lower body weight. Underweight patients were also found to have higher exposure to oral anticoagulants. ${ }^{16}$ The former study was considerably smaller, utilized slightly different definition of complications, and did not analyze MVCs separately. Nevertheless, it was not reported that BSA was comparable to body weight in predicting complications already at that time.

The current study identified low body height to be the most predictive for MVCs, particularly in females. Females of low stature independently of body weight are at higher risk of MVCs, which is in contrast to the common belief and scarce data ${ }^{17}$ that obese patients may have relatively lower levels of anticoagulants, causing less frequent complications. Our high-risk female patients were of low stature, not notably underweight. Of importance is the magnitude of the effect. By every $5 \mathrm{~cm}$ of decrease of body height in females, MVCs rise by $1.3 \%$. On the contrary, in males body height failed to characterize patients with MVCs, while BSA was univariately associated with MVCs. A similar relationship of vascular complications to body size was observed in a study by Piper et al. after the coronary interventions. ${ }^{18}$ They found lower stature and BSA $<1.6 \mathrm{~m}^{2}$ (OR: 4.4, 95\% CI: $3.32-5.96, \mathrm{P}=0.001$ ) as a predictor of vascular complications, however, no gender differences were analyzed.

We may only speculate about higher MVC in females and relationship to body size. Although female gender itself did not numerically play a role in a rate of MVCs, this is not by far a proof of absent impact. First, it has been shown that the pharmacokinetic profile of heparin is different in females as compared to males with a higher preponderance of women to increased values of ACT after administration of the same loading dose of a drug. ${ }^{17}$ Body size and composition likely influence the plasma level of heparin. Winkle et al. ${ }^{19}$ showed that with the increased level of heparin anticoagulation, vascular and hemorrhagic complications increased linearly from 1.62 to $5.55 \%$. Second, some studies described the differences in femoral vascular anatomy between men and women, with a smaller size of both artery and vein and the femoral artery and circumflex branches running very close and often overlapping the femoral vein in females. ${ }^{20,21}$ Sharma et al. demonstrated that one of the predictors of the MVC was the use of more than 4 vascular accesses in the groin. ${ }^{22}$ In UK prospective PCI registry, the use of larger size femoral access sheaths was associated with an increased risk of bleeding.

US-guided venipuncture

Among predictors of MVCs, US - guidance for venous access plays a paramount role. Sharma et al. documented a significant decrease in MVCs using US guidance. ${ }^{22}$ In another analysis on 499 elderly women, vascular complications occurred less often in the US-guided group. ${ }^{23}$ In our former randomized trial, USguided venipuncture did not demonstrate a significant benefit in respect to MVCs, probably due to low event rate, however, all intra-procedural measures were in favor of the US-guided approach. ${ }^{24}$ In a meta-analysis by Sobolev et al., the use of real-time US-guidance decreased access-related bleeding and life-threatening vascular complications, thought the number needed to treat was quite large. ${ }^{25}$ Another larger meta-analysis demonstrated that the use of US-guidance for vascular access in EP procedures reduces the risk of MVC by $71 \%$ compared with the standard anatomical approach. ${ }^{26}$ Moreover US guidance significantly reduced puncture time and inadvertent arterial puncture. ${ }^{26}$ Our results confirmed favorable outcome in respect to MVCs only in males, females do not seem to benefit from US-guidance. This may support the hypothesis about gender-related differences in the pharmacokinetic profile of heparin and dependence of plasma heparin level on body size and composition or size of the sheaths in relation to the vessel diameter rather than the arrangement of the vessels.

Complication rate during the study period

In this study, we revealed a mean reduction of MVCs by $1.7 \%$ during the study period embracing almost 13 
years. Within this time frame, several changes in procedural workflow occurred. These include the switch to uninterrupted oral anticoagulation without bridging with low molecular weight heparin, increasing use of direct oral anticoagulants, US-guided venipuncture or the use of "Z"-stitch for venous access site closure as examples. Only US-guided venipuncture led to the reduction of MVCs in males, other measures have not resulted in the tangible reduction of MVCs. In contrast to the vast clinical knowledge of lower bleeding rate with the use of uninterrupted oral anticoagulation in $\mathrm{CA}$ for $\mathrm{AF}^{27}$, no $\mathrm{MVC}$ reduction was observed in our analysis. Unfortunately, we do not have the data on the proportion of direct oral anticoagulants and this may be one of the factors. Generally, low complication rate may be another factor. Besides these changes, individual and cumulative operator experience also played a role, but this cannot be easily quantified and analyzed.

Implications

Our observations may have implications for clinical practice. First, because a body size contributes to the level of intraprocedural anticoagulation, we may reconsider a lower level of ACT target in females, especially in those with lower stature. In this respect, it has to be emphasized that current recommendations of intraprocedural ACT between 300 to $350 \mathrm{~s}$ are largely based on intracardiac echocardiographic observation of small thrombi with ACT values around $250 \mathrm{~s}$ in the era of interrupted periprocedural oral anticoagulation and not on actual clinical events. Some observations documented the safety of the AF ablation procedure with lower ACT levels (even below $210 \mathrm{~s}$ ). ${ }^{17}$ Second, given a higher rate of MVCs in smaller women, we may speculate that using a smaller diameter of vascular sheaths could decrease the risk of MVCs.

\section{Limitations}

The study has several limitations. First, it is a retrospective analysis and during the study period, many changes occurred in the procedural strategy and anticoagulation treatment. Consequently, some relevant factors, e.g. bridging strategy, details on non-interruption schemes, types of anticoagulants, experience of operators, fellows in training engagement, were not collected systematically to be included in the multivariate analysis. Second, the relatively low rate of MVCs in our center results in a relatively low power of the study to identify the effect of other procedural and anticoagulation factors. Third, conclusions derived from multivariate analysis do not necessarily reflect the causality, so that we cannot exclude the impact of gender itself on a rate of MVCs. Finally, although quite large, it still reflects single-center experience.

\section{Conclusions}

MVCs are twice as often in females than in males following CA for AF. This difference is driven by lower body size in females. We did not identify any direct impact of gender per se on the rate of MVCs. Low body height in females and advanced age in males were independent risk factors of MVCs. US-guided venipuncture lowered the MVC rate in males. These observations may have implications for the strategy of periprocedural anticoagulation, implementation of US-guidance for venous access, and for the selection of vascular sheaths.

\section{References:}

1. Zoni-Berisso M, Lercari F, Carazza T, Domenicucci S. Epidemiology of atrial fibrillation: European perspective. Clin Epidemiol 2014;6:213-20.

2. Steinbeck G, Sinner MF, Lutz M, Muler-Nurasyid, Kaab S, Reinecke H. Incidence of complications related to catheter ablation of atrial fibrillation and atrial flutter: a nationwide in-hospital analysis of administrative data for Germany in 2014. Eur Heart J 2018;39:4020-29.

3. Stabile G, Bertaglia E, Pappone C, Themistoclakis S, Tondo C, Zorzi Aet al . Influence of age and gender on complications of catheter ablation for atrial fibrillation. J Atr Fibrillation 2015;7 :1197.

4. Shah RU, Freeman JV, Shilane D, Wang PJ, Go AS, Hlatky MA. Procedural complications, rehospitalizations, and repeat procedures after catheter ablation for atrial fibrillation. J Am Coll Cardiol 2012;59:143-49.

5. Calkins H, Hindricks G, Cappato R, Kim YH, Saad EB, Aguinaga Let al . 2017 HRS/EHRA/ECAS/APHRS/SOLAECE expert consensus statement on catheter and surgical abla- 
tion of atrial fibrillation. Europace 2018;20:e1-e160.

6. Aldhoon B, Wichterle D, Peichl P, Čihák R, Kautzner J. Complications of catheter ablation for atrial fibrillation in a high-volume centre with the use of intracardiac echocardiography. Europace 2013;15:2432.

7. Cheung JW, Cheng EP, Wu X, Yeo I, Christos PJ, Kamel H et al . Sex-based differences in outcomes, 30-day readmissions, and costs following catheter ablation of atrial fibrillation: the United States Nationwide Readmissions Database 2010-14. Eur Heart J 2019;40:3035-43.

8. Kaiser DW, Fan J, Schmitt S, Than CT, Ullal AJ, Piccini JP et al . Gender differences in clinical outcomes after catheter ablation of atrial fibrillation. JACC Clin Electrophysiol 2016;2:703-10.

9. Spragg DD, Dalal D, Cheema A, Scherr D, Schilukuri K, Cheng A et al. Complications of catheter ablation for atrial fibrillation: Incidence and predictors. J Cardiovasc Electrophysiol 2008;19:627-31.

10. Patel D, Mohanty P, Di Biase L, Sanchez JE, Shaheen MH, Burkhardt JDet al. Outcomes and complications of catheter ablation for atrial fibrillation in females. Heart Rhythm 2010;7:167-72.

11. Zhang XD, Tan HW, Gu J, Jiang WF, Zhao L, Wang YL et al.Efficacy and safety of catheter ablation for long-standing persistent atrial fibrillation in women. Pacing Clin Electrophysiol 2013;36:1236-44.

12. Dagres N, Hindricks G, Kottkamp H, Sommer P, Gaspar T, Bode K et al . Complications of atrial fibrillation in 1000 consecutive procedures: still cause for concern? J Cardiovasc Electrophysiol 2009;20:1014-9.

13. Gupta A, Perera T, Ganesan A, Sullivan T, Lau DH, Roberts-Thomson KCet al . Complications of catheter ablation of atrial fibrillation: a systematic review. Circ Arrhythm Electrophysiol 2013;6:10828.

14. Argulian E, Patel AD, Abramson JL, Kulkarni A, Champney K, Palmer Set al. Gender differences in short-term cardiovascular outcomes after percutaneous coronary interventions. Am J Cardiol 2006;98:48-53.

15. Sharma V, Wilson W, Smith W, McEntegart M, Oldroyd K, Sidik N et al. Comparison of characteristics and complications in men versus women undergoing chronic total occlusion percutaneous intervention. Am J Cardiol 2017;119:535-41.

16. McCaughan GJB, Favaloro EJ, Psalic L, Curnow J. Anticoagulation at the extremes of body weight: choices and dosing. Expert Rev Hematol 2018;11:817-28.

17. Campbell NR, Hull RD, Brant R, Hogan DB, Pineo GF, Raskob GE. Different effects of heparin in males and females. Clin Invest Med 1998;21:71-8.

18. Piper WD, Malenka DJ, Ryan TJ jr, Shuhbrooks SJ jr, O'Connor GT, Robb JF el al . Predicting vascular complications in percutaneous coronary interventions. Am Heart J 2003;145:1022-9.

19. Winkle RA, Mead RH, Engel G, Patrawala RA. Safety of lower activated clotting times during atrial fibrillation ablation using open irrigated tip catheters and a single transseptal puncture. Am J Cardiol 2011;107: 704-8

20. Uhl JF, Gillot C, Chahim M. Anatomical variations of the femoral vein. J Vasc Surg 2010;52:714-9.

21. Czyzewska D, Ustymowicz A, Kowalewski R, Zurada A, Krejza J. Cross-sectional area of the femoral vein varies with leg position and distance from the inguinal ligament. Plos One 2017;12: 1-11.

22. Sharma P, Padala S, Gunda S, Koneru JN, Ellenbogen KA. Vascular complications during catheter ablation of cardiac arrhythmias: a comparison between vascular ultrasound guided access and conventional vascular access. J Cardiovasc Electrophysiol. 2016;27:1160-6.

23. Mohanty S, Trivedi C, Gianni C, Burkhardt J, Sanchez R et al. Real-time ultrasound guidance for venous access reduces vascular complications in women aged 75 years or older undergoing catheter ablation for atrial fibrillation under uninterrupted anticoagulation. Eur Heart J 2018;39:suppl 1,P5755

24. Yamagata K, Wichterle D, Roubíček T, Jarkovský P, Sato J Kogure T et al. Ultrasound-guided versus conventional femoral venipuncture for catheter ablation of atrial fibrillation: a multicentre randomized efficacy and safety trial (ULTRA-FAST trial). Europace 2018;7:1107-1114.

25. Sobolev M, Shiloh AL, Di Biase L, Slovut DP. Ultrasound-guided cannulation of the femoral vein in electrophysiological procedures: a systematic review and meta-analysis. Europace 2017;5:850-855.

26. Kupó P, Pap R, Sághy L, Tényi D, Bálint A, Debreceni D et al. Ultrasound guidance for femoral 
venous access in electrophysiology procedures-systematic review and meta-analysis. J Interv Card Electrophysiol 2019;10:s10840-019-00683.

27. Yang P, Wang C, Ye Y, Huang T, Yang S, Shen W et al. Interrupted or uninterrupted oral anticoagulants in patients undergoing atrial fibrillation ablation. Cardiovasc Drugs Ther 2020;34:371-381

Table 1. Baseline characteristics of the study population

\begin{tabular}{lllll}
\hline & Total population & Males & Females & P value \\
\hline & $\mathrm{N}=4734$ & $\mathrm{~N}=3222$ & $\mathrm{~N}=1512$ & \\
Age (years) & $60 \pm 10$ & $58.7 \pm 10.1$ & $63.3 \pm 8.7$ & $<0.0001$ \\
Males (\%) & 71 & - & - & \\
Paroxysmal AF (\%) & 65 & 63.1 & 68.7 & $<0.0001$ \\
Body weight (kg) & $90.7 \pm 16.6$ & $95.6 \pm 14.8$ & $80.4 \pm 15.4$ & $<0.0001$ \\
Body height (cm) & $176.4 \pm 9.6$ & $180.9 \pm 7.3$ & $166.8 \pm 6.4$ & $<0.0001$ \\
Body mass index (kg/m ${ }^{2}$ ) & $29.1 \pm 4.6$ & $29.2 \pm 4.2$ & $28.9 \pm 5.3$ & 0.02 \\
Body surface area (m $\left.{ }^{2}\right)$ & $2.07 \pm 0.22$ & $2.16 \pm 0.18$ & $1.89 \pm 0.18$ & $<0.0001$ \\
CHA $_{2}$ DS $_{2}$-VASc score & $1.8 \pm 1.4$ & $1.4 \pm 1.2$ & $2.6 \pm 1.2$ & $<0.0001$ \\
LV ejection fraction (\%) & $56 \pm 8$ & $55.7 \pm 8.2$ & $57.4 \pm 6.5$ & $<0.0001$ \\
Left atrial diameter (mm) & $42.5 \pm 5.6$ & $43.4 \pm 5.5$ & $40.6 \pm 5.5$ & $<0.0001$ \\
Hypertension (\%) & 60.6 & 58.5 & 64.9 & $<0.0001$ \\
Heart failure (\%) & 16.5 & 18.0 & 13.3 & $<0.0001$ \\
Diabetes mellitus (\%) & 12.3 & 12.5 & 11.9 & 0.53 \\
Previous stroke/TIA (\%) & 6.1 & 5.6 & 7.2 & 0.03 \\
CAD (\%) & 8.6 & 9.6 & 6.3 & $<0.0001$ \\
eGFR (CKD-EPI, ml/min) & $76.9 \pm 17.3$ & $79.6 \pm 16.5$ & $71.1 \pm 17.5$ & $<0.0001$ \\
Procedure time (min) & $216 \pm 71$ & $218 \pm 72$ & $210 \pm 68$ & $<0.0001$ \\
Radiofrequency time (min) & $44.2 \pm 21.6$ & $45.5 \pm 22.2$ & $41.4 \pm 19.8$ & $<0.0001$ \\
Substrate ablation (\%) & 39.2 & 37.9 & 42.0 & 0.007 \\
CTI ablation (\%) & 22.1 & 22.0 & 22.2 & 0.83 \\
Repeated ablation (\%) & 26.8 & 27.3 & 25.8 & 0.29 \\
Robotic ablation (\%) & 9.1 & 9.7 & 8.0 & 0.07 \\
Noninterrupted OAC (\%) & 56.0 & 54.8 & 58.5 & 0.02 \\
"Z" stitch (\%) & 46.9 & 45.9 & 48.9 & 0.05 \\
US-guided venipuncture (\%) & 26.0 & 25.5 & 27.0 & 0.28 \\
\hline
\end{tabular}

Indices are shown per procedure as mean \pm standard deviation or proportion in percentages and compared for male and females. AF, atrial fibrillation; CAD, coronary artery disease; CKD-EPI, Chronic Kidney Disease Epidemiology Collaboration; CTI, cavotricuspid isthmus; eGFR, estimated glomerular filtration rate; LV, left ventricle; OAC, oral anticoagulation; TIA, transient ischemic attack; US, ultrasound.

Table 2. Comparison of patient subgroups with and without major vascular complications (separately for males and females)

\begin{tabular}{llllll}
\hline & MVCs in MALES & MVCs in MALES & MVCs in MALES & MVCs in FEMALES & MVCs \\
\hline & present & absent & $\mathrm{P}$ & present & absent \\
& $\mathrm{N}=58$ & $\mathrm{~N}=3164$ & & $\mathrm{~N}=54$ & $\mathrm{~N}=14$ \\
Age (years) & $61.8 \pm 8.7$ & $58.6 \pm 10.1$ & 0.02 & $66.2 \pm 5.6$ & $63.2 \pm$ \\
Paroxysmal AF (\%) & 48.3 & 63.4 & 0.02 & 64.8 & 68.8 \\
Body weight $(\mathrm{kg})$ & $92.0 \pm 14.0$ & $95.6 \pm 14.8$ & 0.06 & $78.6 \pm 16.6$ & $80.4 \pm$ \\
Body height $(\mathrm{cm})$ & $180 \pm 7$ & $181 \pm 7$ & 0.19 & $164 \pm 6$ & $167 \pm 6$ \\
Body mass index $\left(\mathrm{kg} / \mathrm{m}^{2}\right)$ & $28.5 \pm 4.1$ & $29.2 \pm 4.2$ & 0.20 & $29.2 \pm 5.6$ & $28.8 \pm$
\end{tabular}




\begin{tabular}{llllll}
\hline & MVCs in MALES & MVCs in MALES & MVCs in MALES & MVCs in FEMALES & MVCs \\
\hline Body surface area $\left(\mathrm{m}^{2}\right)$ & $2.11 \pm 0.17$ & $2.16 \pm 0.18$ & 0.05 & $1.84 \pm 0.19$ & $1.89 \pm$ \\
CHA $_{2}$ DS $_{2}$-VASc score & $1.7 \pm 1.2$ & $1.4 \pm 1.2$ & 0.09 & $2.8 \pm 1.0$ & $2.6 \pm 1$ \\
LV ejection fraction (\%) & $55.9 \pm 7.3$ & $55.7 \pm 8.2$ & 0.85 & $56.1 \pm 8.5$ & $57.4 \pm$ \\
Left atrial diameter (mm) & $43.9 \pm 5.5$ & $43.4 \pm 5.5$ & 0.50 & $41.9 \pm 5.0$ & $40.5 \pm$ \\
Hypertension (\%) & 63.8 & 58.4 & 0.41 & 74.1 & 64.5 \\
Heart failure (\%) & 17.2 & 18.0 & 0.87 & 13.0 & 13.3 \\
Diabetes mellitus (\%) & 20.7 & 12.4 & 0.06 & 7.4 & 12.1 \\
Previous stroke/TIA (\%) & 3.4 & 5.6 & 0.48 & 7.4 & 7.2 \\
CAD (\%) & 13.8 & 9.5 & 0.28 & $72.0 \pm 17.9$ & $71.0 \pm$ \\
eGFR (CKD-EPI, ml/min) & $76.3 \pm 17.5$ & $79.6 \pm 16.5$ & 0.14 & $227 \pm 77$ & $209 \pm 1.5 \pm$ \\
Procedure time (min) & $239 \pm 74$ & $218 \pm 71$ & 0.03 & $39.9 \pm 20.2$ & $41.5 \pm 1.9$ \\
Radiofrequency time (min) & $49.9 \pm 25.1$ & $45.4 \pm 22.1$ & 0.13 & 22.2 & 22.2 \\
Substrate ablation (\%) & 48.3 & 37.7 & 0.10 & 24.1 & 25.9 \\
CTI ablation (\%) & 13.8 & 22.1 & 0.13 & 7.4 & 8.0 \\
Repeated ablation (\%) & 25.9 & 27.3 & 0.81 & 51.9 & 58.7 \\
Robotic ablation (\%) & 14.0 & 9.6 & 0.26 & 44.4 & 29.1 \\
Noninterrupted OAC (\%) & 46.6 & 55.0 & 0.14 & 25.9 & 27.0 \\
"Z" stitch (\%) & 36.2 & 46.0 & 0.007 & & \\
US-guided venipuncture (\%) & 10.3 & 25.8 & & &
\end{tabular}

Indices are shown per procedure as mean \pm standard deviation or proportion in percentages and compared for male and females.

MVC, major vascular complication; other abbreviations as in Table 1.

Table 3. Gender differences in the rate of major vascular complications (subcategories)

\begin{tabular}{llllll}
\hline Complication type & Males & Males & Females & Females & P value \\
\hline & $\mathrm{N}$ & $\%$ & $\mathrm{~N}$ & $\%$ & \\
Groin hematoma & 23 & 0.71 & 35 & 2.31 & $<0.0001$ \\
Jugular hematoma & 3 & 0.09 & 2 & 0.13 & $\mathrm{NS}$ \\
Retroperitoneal/pelvic bleeding & 1 & 0.03 & 2 & 0.13 & $\mathrm{NS}$ \\
Groin pseudoaneurysm & 15 & 0.47 & 15 & 0.99 & $\mathrm{NS}$ \\
Groin arteriovenous fistula & 9 & 0.28 & 3 & 0.20 & $\mathrm{NS}$ \\
Hemothorax & 3 & 0.09 & 0 & 0 & $\mathrm{NS}$ \\
Deep vein thrombosis & 3 & 0.09 & 0 & 0 & $\mathrm{NS}$ \\
Sheath rupture/embolization & 2 & 0.06 & 0 & 0 & $\mathrm{NS}$ \\
\hline
\end{tabular}

In case of duplicity of major vascular complication, both were considered. This occurred in 1 procedure in male and 3 procedures in females.

Table 4. Predictors of major vascular complications (MVCs) assessed separately for both genders

\begin{tabular}{lllll}
\hline & Males & Males & Males & Males \\
\hline & Univariate regression & Univariate regression & Univariate regression & Multivariate \\
& & & $\mathbf{R}=\mathbf{0 . 0 6 6 , \mathbf { P }}$ \\
FACTOR & SLOPE \pm SEM & $\mathrm{R}$ & $\mathrm{P}$ & SLOPE \pm SEM \\
Age (years) & $0.056 \pm 0.023 \%$ & 0.04 & 0.02 & $0.062 \pm 0.023 \%$
\end{tabular}




\begin{tabular}{lllll}
\hline & Males & Males & Males & Males \\
\hline Body weight $(\mathrm{kg})$ & $-0.029 \pm 0.016 \%$ & 0.03 & 0.06 & $\mathrm{n} / \mathrm{s}$ \\
Body height $(\mathrm{cm})$ & $-0.042 \pm 0.032 \%$ & 0.02 & 0.19 & $\mathrm{n} / \mathrm{a}$ \\
Body surface area $\left(\mathrm{m}^{2}\right)$ & $-2.61 \pm 1.33 \%$ & 0.03 & 0.05 & $\mathrm{n} / \mathrm{s}$ \\
Diabetes mellitus $(1 / 0)$ & $1.34 \pm 0.71 \%$ & 0.03 & 0.06 & $\mathrm{n} / \mathrm{s}$ \\
$\mathrm{CHA}_{2} \mathrm{DS}_{2}$-VASc score & $0.39 \pm 0.21 \%$ & 0.03 & 0.06 & $\mathrm{n} / \mathrm{s}$ \\
Paroxysmal AF $(1 / 0)$ & $-1.15 \pm 0.49 \%$ & 0.04 & 0.02 & $\mathrm{n} / \mathrm{s}$ \\
Procedure time (min) & $0.007 \pm 0.003 \%$ & 0.04 & 0.03 & $\mathrm{n} / \mathrm{s}$ \\
US-guided venipuncture & $-1.44 \pm 0.54 \%$ & 0.05 & 0.007 & $-1.55 \pm 0.54 \%$ \\
Year of procedure & $-0.154 \pm 0.071 \%$ & 0.04 & 0.03 & $\mathrm{n} / \mathrm{s}$ \\
\hline
\end{tabular}

Linear regression was used. Only factors are shown that were univariately associated $(\mathrm{P}<0.1)$ with MVCs either in males or females. AF, atrial fibrillation; $\mathrm{n} / \mathrm{a}$, factor did not enter the multivariate model due to univariate $\mathrm{P}>0.1 ; \mathrm{n} / \mathrm{s}$, factor was not significant in multivariate model (stepwise forward method); SEM, standard error of the mean; SLOPE, change of MVC rate (\%) per unit change of factor; R, regression coefficient; US, ultrasound.

\section{Figure legends}

Figure 1. Timeline of major vascular complication rate.

Box and whiskers represent standard error of mean and $95 \%$ confidence interval, respectively.

Figure 2. Plot of major vascular complication rate in quartiles of age and categorized by gender.

Box and whiskers represent standard error of mean and $95 \%$ confidence interval, respectively. There is a trend towards higher event rate in females compared to males in 3 rd and 4th quartile $(\mathrm{P}=0.09$ and $\mathrm{P}=$ 0.06 , respectively).

Figure 3. Plot of major vascular complication rate in quartiles of body height (left) and weight (right) categorized by gender. Box and whiskers represent standard error of mean and $95 \%$ confidence interval, respectively.

Figure 4. XYZ contour chart of major vascular complication rate stratified by body height and weight. Separate plots are provided for females (left) and males (right). Height and weight axes are scaled to cover the range of $10^{\text {th }}$ to $90^{\text {th }}$ percentile of particular variable as assessed separately for both genders.

\section{Figure 1.}




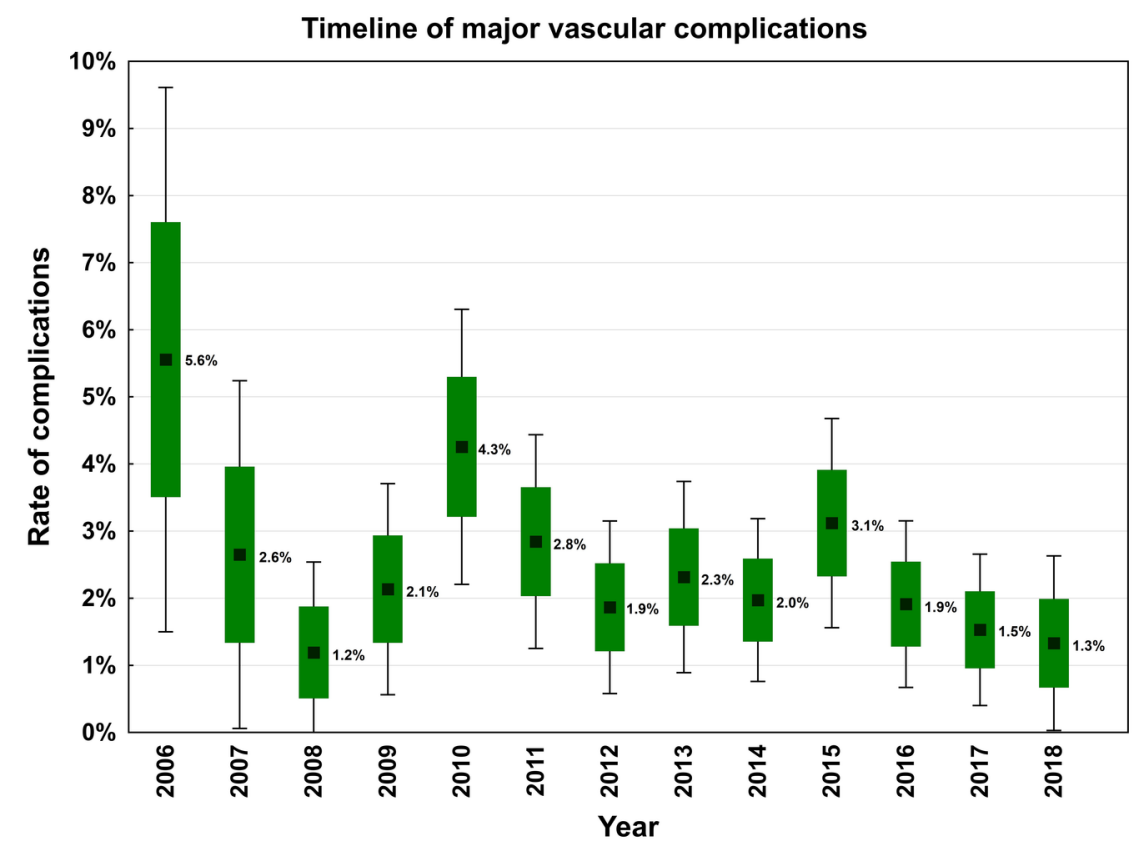

Figure 2.

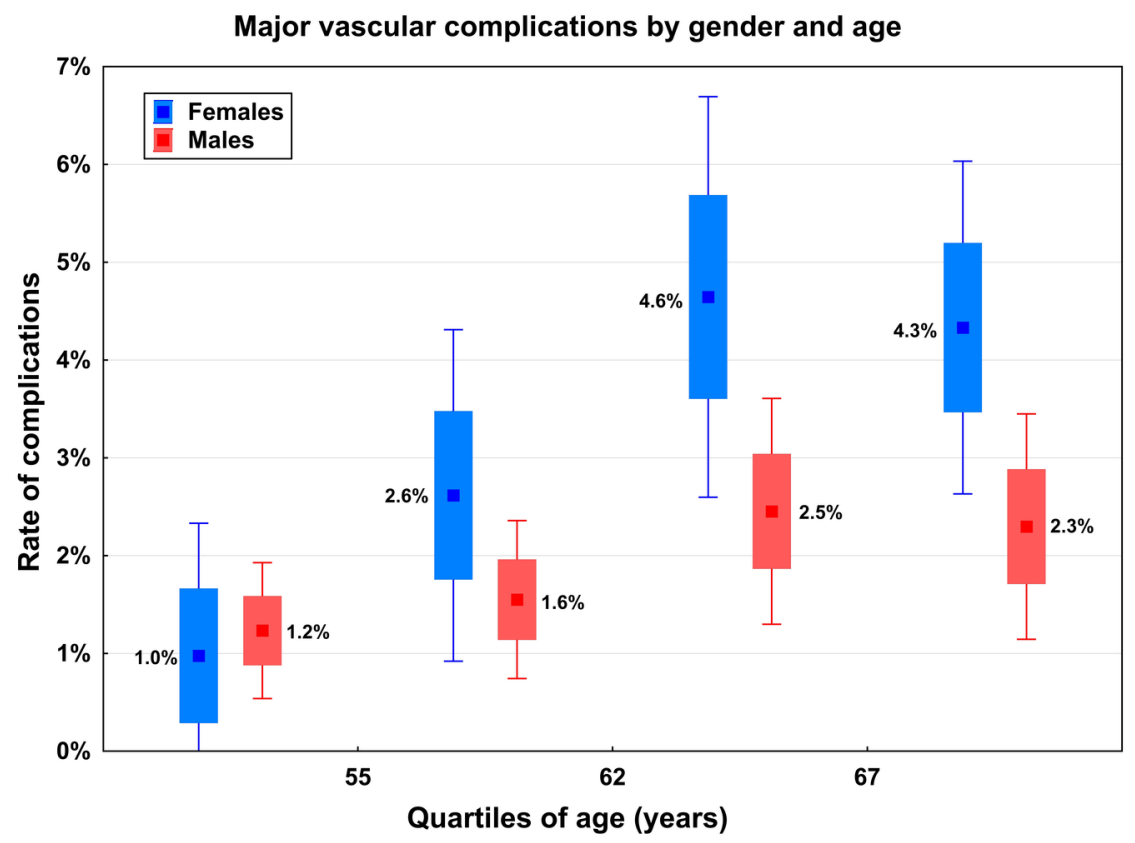

Figure 3. 
Gender differences in major vascular complications

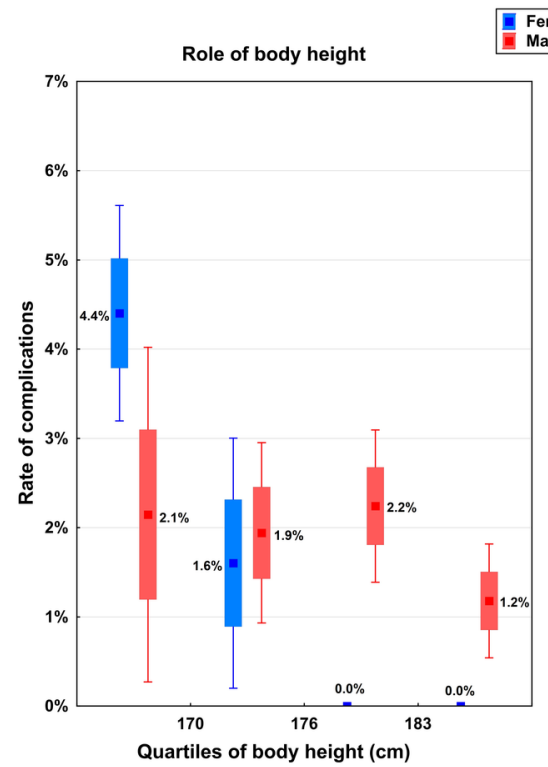

\begin{tabular}{l}
$\begin{array}{l}\text { Females } \\
\text { Males }\end{array}$ \\
\hline
\end{tabular}

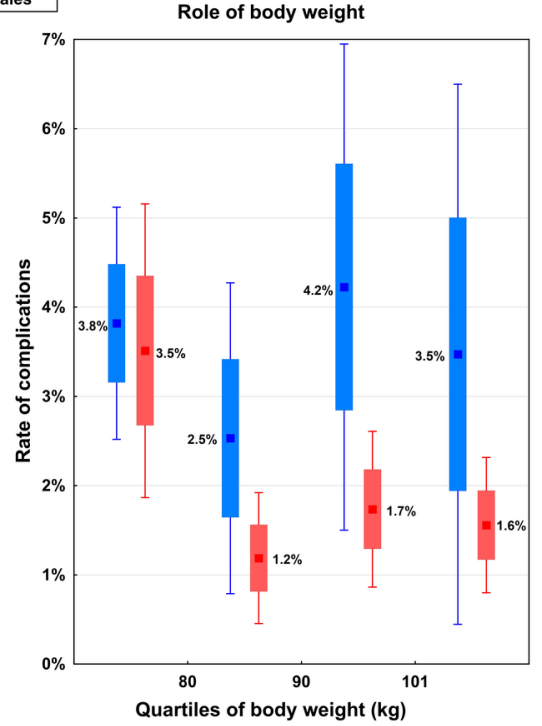

Figure 4 .

Major vascular complications by body height and weight

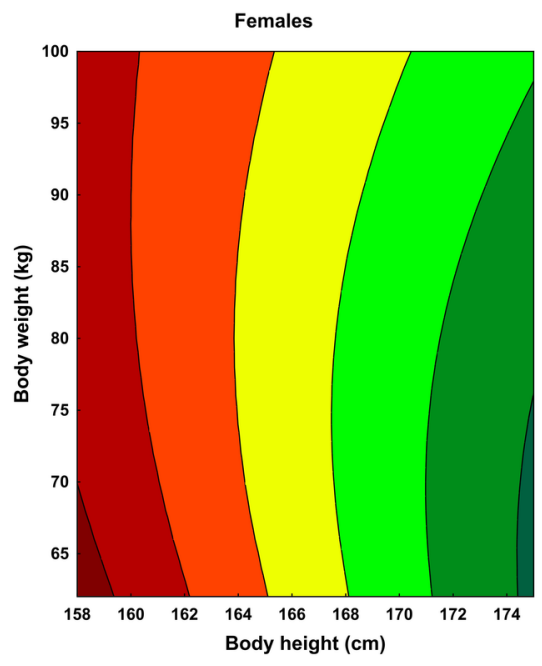

Complication rate:

$>6.0 \% \quad<6.0 \% \quad<5.0 \% \quad<4.0 \%$

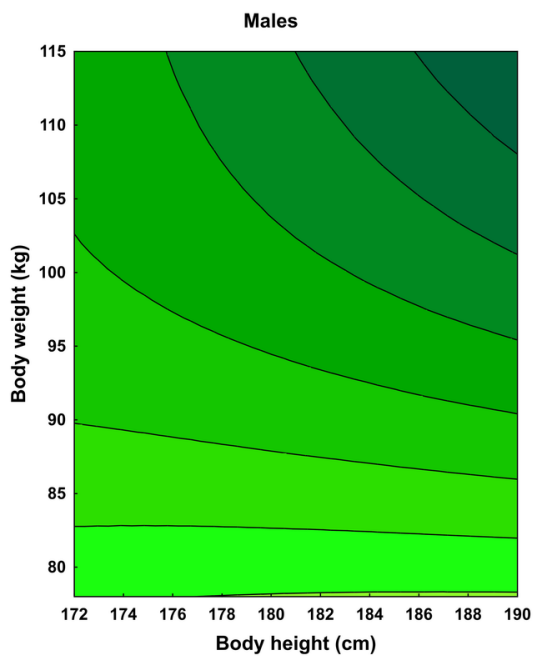

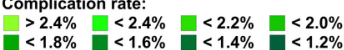

